\title{
Reactor antineutrino physics with DANSS experiment
}

\author{
Igor Alekseev*† \\ Alikhanov Institute for Theoretical and Experimental Physics NRC "KI"; \\ National Research Nuclear University MEPhI; \\ Moscow Institute of Physics and Technology \\ E-mail: igor.alekseev@itep.ru
}

\begin{abstract}
DANSS is a solid state scintillation detector of reactor antineutrino, placed just below $3.1 \mathrm{GW}$ industrial light water reactor of Kalininskaya Nuclear Power Plant about 350 km NW from Moscow. A cubic meter sensitive volume of the detector is formed by 2500 scintillator strips with individual SiPM readout. Groups of 50 strips are also readout by conventional PMTs. Reactor antineutrinos are detected by inverse beta-decay (IBD). The sensitive volume has passive shielding of copper $(5 \mathrm{~cm})$, borated polyethylene (two layers of $8 \mathrm{~cm}$ ), lead $(5 \mathrm{~cm})$ and active shielding of double layer scintillation counters. The position below reactor provides overburden about 50 m.w.e. A movable platform of the detector allows to change its distance to the reactor from 10.7 to $12.7 \mathrm{~m}$ (center to center). 5000 IBD events are detected daily in the position closest to the reactor.

Ratio of the antineutrino spectra at different distances measured with the DANSS detector is sensitive to neutrino oscillations to sterile neutrinos in the most interesting region of the oscillation parameters space (delta $\mathrm{m}$ squared $1 \mathrm{eV}^{2}$ ), where the effect is suspected by Gallium and Reactor antineutrino anomalies. The DANSS collaboration presents results of 1.5 year detector running with three positions $(10,7,11.7$ and $12.7 \mathrm{~m})$ cycled weekly. The talk covers sterile neutrino search results as well as analysis details.
\end{abstract}

The 39th International Conference on High Energy Physics (ICHEP2018)

4-11 July, 2018

Seoul, Korea

* Speaker.

${ }^{\dagger}$ for the DANSS collaboration 
Neutrinos are the most mysterious particles of the Standard model. It is well established now that there are three flavors of "usual" neutrinos and that they can transform to each other via oscillation mechanism [1]. Neutrino oscillations are now well measured in many appearance and disappearance experiments. Nevertheless not all results of these experiments could be explained in the framework with three "usual" neutrinos. There are several "anomalies" which require short length oscillations with $\Delta m^{2} \sim 1 \mathrm{eV}$ [2]. This explanation introduces a new neutrino flavor, which can not participate in the weak interaction and manifests itself only in oscillations.

Industrial reactors are the most powerful sources of terrestrial neutrinos. DANSS detector was designed of solid state scintillator, so it is possible to place it just below a reactor with antineutrino flux about $5 \cdot 10^{13} \mathrm{~cm}^{-2} \mathrm{~s}^{-1}$. The detector is placed on a movable platform, so we can measure antineutrino spectrum at different distances using one and the same detector. This allows us to search for short range neutrino oscillations in the evolution of the spectra and avoid all systematic uncertainties connected to neutrino spectra calculations, detector efficiency calculations and so on.

DANSS is a fine segmented solid state scintillation detector [3]. The sensitive volume is formed by 2500 one meter long strips $1 \times 4 \mathrm{~cm}^{2}$ in cross section. The strips are covered by reflective layer with gadolinium oxide admixture and have dual readout. Each strip is individually read by SiPM and modules of 50 strips are read by conventional PMTs. The cubic meter sensitive volume is surrounded by multilayer passive shielding of $5 \mathrm{~cm}$ copper, $8 \mathrm{~cm}$ borated polyethylene, $5 \mathrm{~cm}$ lead and another $8 \mathrm{~cm}$ of borated polyethylene. A double layer active veto of scintillator counters covers the detector from 5 sides with an exception of the bottom. The assembly and electronics are placed on a movable platform which allows to move detector vertically for $2 \mathrm{~m}$. In the upper position the center of the detector is $10.7 \mathrm{~m}$ from the center of the core of $3.1 \mathrm{GW}_{t h}$ light water industrial reactor WWER1000 of Kalinin Nuclear Power Plant. The overburden above the DANSS hall is about $50 \mathrm{~m}$ w.e., providing about 6 times cosmic muon flux suppression.

Detection of the antineutrinos is performed in the inverse beta-decay (IBD) reaction:

$$
\bar{v}_{e}+p \rightarrow e^{+}+n, \quad E_{e^{+}}=E_{\bar{v}}-1.80 \mathrm{MeV},
$$

where the positron takes the most of the kinetic energy. The positron stops in the detector material. Its ionization track and annihilation gammas produce an immediate response in the detector. The neutron is slowered down to the thermal energies and after some drift in the detector is captured by gadolinium, producing about $8 \mathrm{MeV}$ gamma flash. So the neutron signal comes several tens of microseconds delayed.

DANSS DAQ is based on 12 bit 125 MSPS waveform digitizers specially designed for this experiment [4]. Trigger condition is formed by instant sum of $0.7 \mathrm{MeV}$ in 50 PMTs reading the sensitive volume of the detector or of $4 \mathrm{MeV}$ in 40 PMTs reading veto counters. On the trigger $512 \mathrm{~ns}$ waveforms are written for all non-zero channels. The calibration is done continuously using ionization energy loss by cosmic muons passing the setup [5]. It was checked with radioactive sources ${ }^{22} \mathrm{Na},{ }^{60} \mathrm{Co}$ and ${ }^{248} \mathrm{Cm}$ (fig. 1).

Data analysis is performed in several stages [6]. At the first stage all signal waveforms are analyzed, energy, time and some other parameters of each hit are stored. At the second stage parameters of each trigger are calculated, including total energy deposited and energy of the continuous ionization cluster considered as a positron track candidate. In the data written an IBD event is present as two separate triggers for positron (prompt) and neutron (delayed) detection. 50 

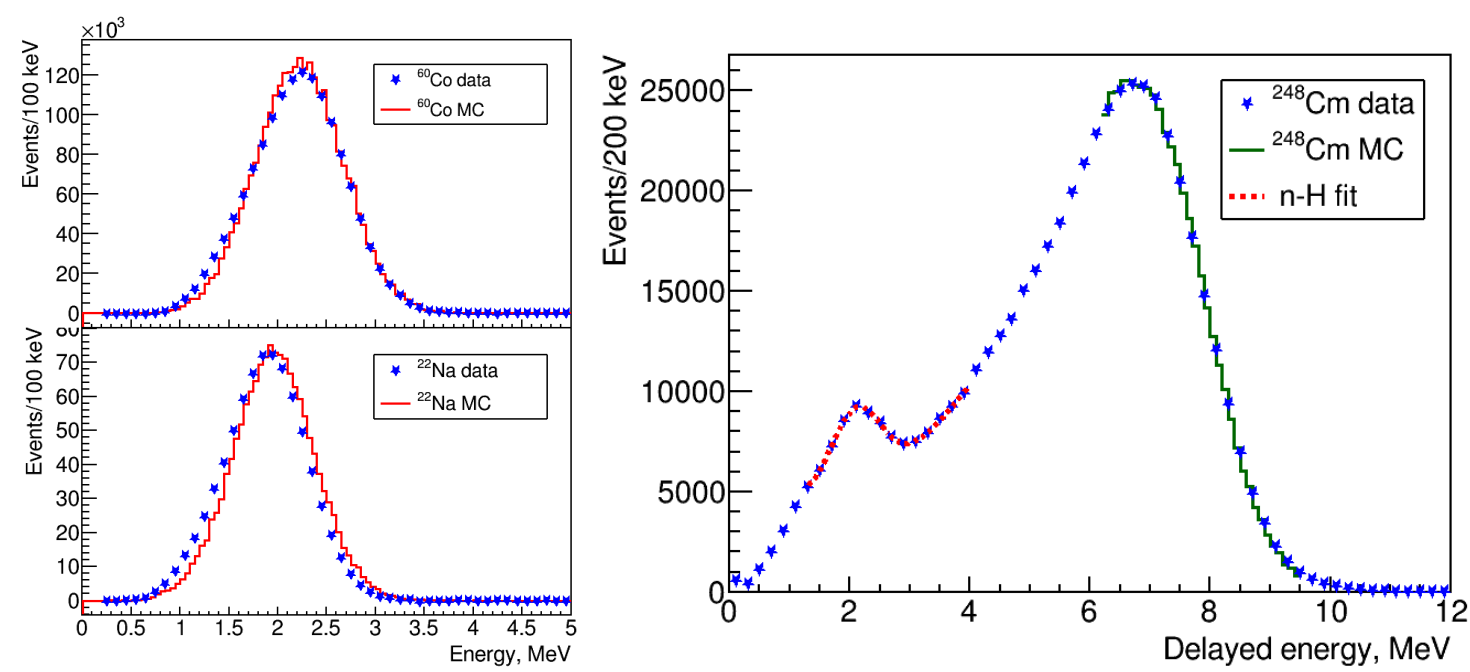

Figure 1: Checking calibration with radioactive sources. ${ }^{60} \mathrm{Co}$ and ${ }^{22} \mathrm{Na}$ are shown in the left panel in comparison to the Geant4 Monte-Carlo simulation of the detector. The right panel demonstrates detector response to neutrons coming from ${ }^{248} \mathrm{Cm}$ fission.

us time window was used for prompt - delayed correlation look up. Uncorrelated background was subtracted using 16 independent time intervals well separated from the signal time window. Figure $2 \mathrm{a}$ illustrates the procedure by a histogram of the time between prompt and delayed events. The signal distribution (green dots) is calculated as a difference between all selected events (blue triangles) and the accidental background (red squares). The resulting positron spectra for three detector positions are shown in fig. 2b. All known backgrounds including contribution from cosmic muons escaped veto and three neighbor reactors are subtracted. The spectrum of the background taken during scheduled reactor shutdown is also shown in the figure.

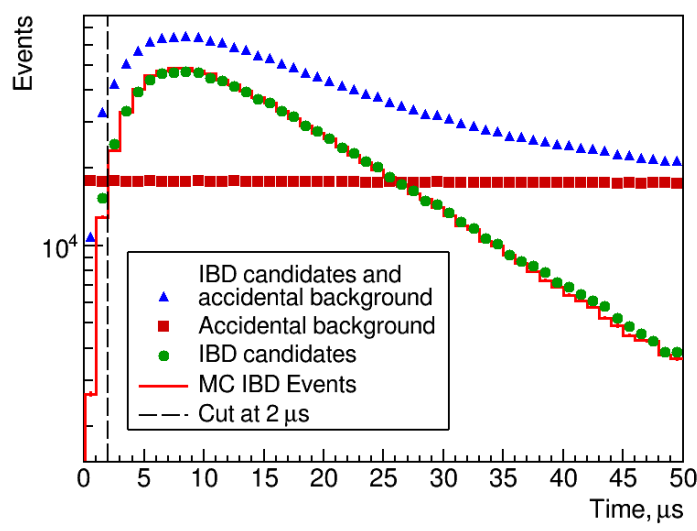

(a)

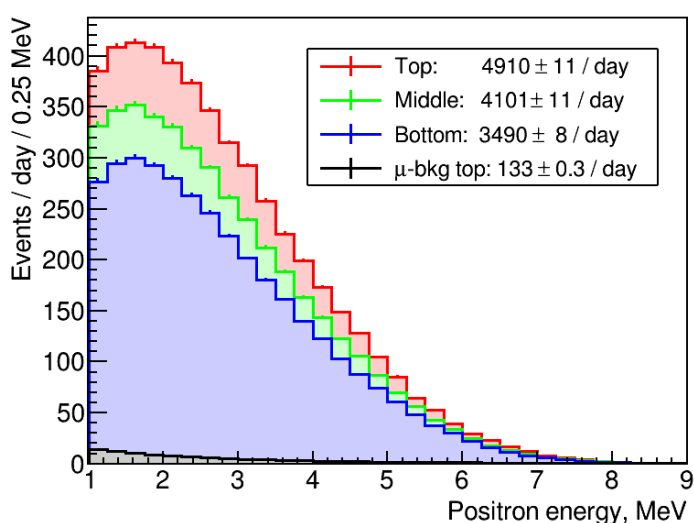

(b)

Figure 2: (a) The time between prompt and delayed events. (b) Positron spectra at the three detector positions.

We use a very conservative Gaussian $\mathrm{CL}_{s}$ method [7] to draw the exclusion region. Several variations of the systematic uncertainty were done. Only points excluded in all variants were put in the final plot. The result is shown in fig. 3 in comparison with sterile neutrino oscillation areas 
expected from gallium and reactor anomalies [8]. The darker area corresponds to $95 \%$ level and the lighter area corresponds to $90 \%$ level. The black star presents the best point by Mention et al. [9]. According to our data this point is excluded at more than 5 sigma level.

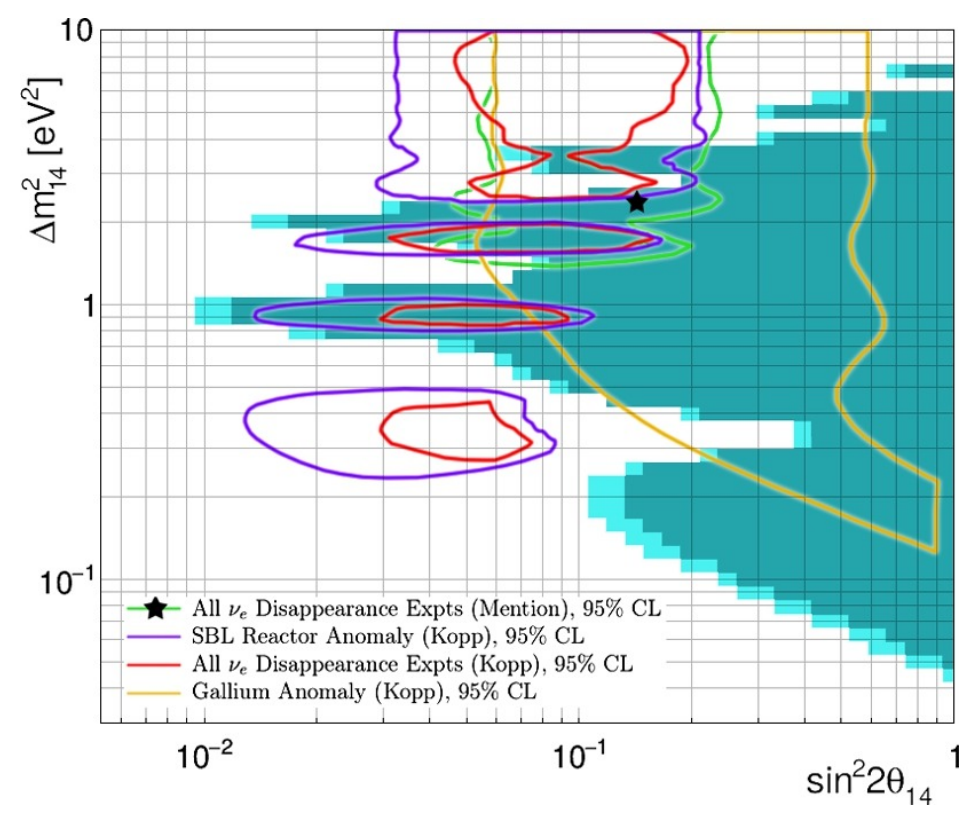

Figure 3: $\mathrm{CL}_{s}$ exclusion region in comparison with allowed regions from neutrino disappearance experiments $[8,9]$.

DANSS collaboration is grateful to the directorates of ITEP and JINR for constant support of this work. The collaboration appreciates the permanent assistance of the KNPP administration and Radiation Safety Department staff. The detector construction was supported by the Russian State Corporation ROSATOM (state contracts H.4x.44.90.13.1119 and H.4x.44.9B.16.1006) and by the Russian Foundation for Basic Research, grant 09-02-00449. The operation and data analysis became possible due to the valuable support from the Russian Science Foundation grant 17-1201145 .

\section{References}

[1] M. Tanabashi et al. (Particle Data Group), Phys. Rev. D 98, 030001 (2018).

[2] K. N. Abazajian et al., Light sterile neutrinos: A white paper, arXiv:1204.5379 [hep-ph].

[3] I. Alekseev et al., JINST 11 (11) (2016) P11011.

[4] I. Alekseev et al., Instr.Exp.Tech. 61 (3) (2018) 349-354.

[5] I. Alekseev et al., Lett.Elem.Part.Atom.Nucl. 15 (3) (2018) 272-283.

[6] I. Alekseev et al., Phys. Lett. B, 787 (2018) 56-63.

[7] X. Qian et al., Nucl.Inst.Meth. A 827 (2016) 63.

[8] J. Kopp, P.A.N. Machado, M. Maltoni and T. Schwetz, J. High Energy Phys. 1305 (2013) 050.

[9] G. Mention, et al. Phys. Rev. D 83 (2011) 073006. 